Nouvelles perspectives en sciences sociales

Revue internationale de systémique complexe et d'études relationnelles

\title{
Vers une théorie de l'interdisciplinarité? Entre unité et diversité
}

\section{Frédéric Darbellay}

Volume 7, numéro 1, octobre 2011

Sur le thème de l'interdisciplinarité

URI : https://id.erudit.org/iderudit/1007082ar

DOI : https://doi.org/10.7202/1007082ar

Aller au sommaire du numéro

Éditeur(s)

Prise de parole

ISSN

1712-8307 (imprimé)

1918-7475 (numérique)

Découvrir la revue

Citer cet article

Darbellay, F. (2011). Vers une théorie de l'interdisciplinarité? Entre unité et diversité. Nouvelles perspectives en sciences sociales, 7(1), 65-87.

https://doi.org/10.7202/1007082ar
Résumé de l'article

L'interdisciplinarité apparaît comme une voie nouvelle dans le développement de l'enseignement et de la recherche universitaires. L'analyse de ses potentialités, mais aussi des obstacles qu'elle rencontre, met en lumière les relations complexes qu'elle entretient avec les disciplines institutionnalisées. L'interdisciplinarité est pensée ici comme un processus d'articulation entre plusieurs disciplines qui ne se résume pas à une simple addition de savoirs hétérogènes. Sans prétendre à une théorie unifiée, qui équivaudrait à la naissance d'un nouveau paradigme, il convient au contraire de prendre en compte la diversité des postures épistémologiques qui contribuent à la co-élaboration du processus interdisciplinaire. 


\title{
Vers une théorie de l'interdisciplinarité? Entre unité et diversité
}

\author{
FrédÉRIC DarbelLAY \\ Institut Universitaire Kurt Bösch, Sion
}

\section{Introduction}

Rien ne nous contraint plus à morceler le réel en compartiments étanches ou en étages simplement superposés correspondant aux frontières apparentes de nos disciplines scientifiques et tout nous oblige au contraire à nous engager dans la recherche des interactions et des mécanismes communs ${ }^{1}$.

Toute interrogation sur l'existence ${ }^{2}$ ou la non-existence d'une 1 théorie générale de l'interdisciplinarité dans le champ des Sciences Humaines et Sociales (SHS) devrait prendre en compte, de manière complémentaire, l'état d'avancement des travaux de réflexion sur l'interdisciplinarité en tant que telle, ainsi que la configuration largement multidisciplinaire de ce champ qui impose des conditions particulières à la mise en dialogue des disciplines. Existe-t-il une "théorie" unifiée de l'interdisciplinarité au sens restreint où elle dicterait un ensemble de propositions

1 Jean Piaget, "L'épistémologie des relations interdisciplinaires ", Bulletin Uni-information, $\mathrm{n}^{\circ} 31$, Genève, 1973, p. 5.

2 Faisant directement écho ici au titre de l'appel à publication pour le présent numéro spécial de la revue NPSS «Y a-t-il une théorie de l'interdisciplinarité en sciences humaines?". 
formelles, logiquement organisées en postulats et universellement validées, à partir desquelles tout chercheur pourrait comprendre, expliquer et interpréter de manière prévisible les pratiques d'articulation entre deux ou plusieurs disciplines dans l'étude de l'homme et de la société?

Si l'on prend acte de la diversité des traditions disciplinaires (sociologie, anthropologie, psychologie, linguistique, études littéraires, sciences politiques, histoire, géographie, sciences de l'éducation, etc.) qui constituent par fragmentation le champ des SHS, elles-mêmes souvent subdivisées en sous-champs disciplinaires, courants ou écoles spécifiques, il semble qu'une théorie unifiée des articulations entre toutes ou parties de ces configurations disciplinaires soit difficilement réalisable. Il n'en demeure pas moins qu'il faut tenter de penser ensemble cette diversité multidisciplinaire et ses possibles articulations, voire intégrations interdisciplinaires. Nous abordons cette problématique en cinq axes interreliés ${ }^{3}$. En partant d'une mise en lumière des divers obstacles qui se dressent face à la mise en œuvre de l'interdisciplinarité (2), leur dépassement est envisagé par la définition d'un cadre théorique et conceptuel dynamique de l'interdisciplinarité (3). Cette manière non dogmatique de définir l'interdisciplinarité répond à la complexité spécifique de l'humain et du social (4) qui réclament en retour une pensée dialogique et non-paradigmatique des liens qui se tissent entre les multiples disciplines qui les prennent pour objet(s) d'étude(s) (5).

\section{Défi de l'interdisciplinarité : des obstacles à dépasser}

Si l'interdisciplinarité fait l'objet de réflexions relativement avancées sur les plans épistémologique, conceptuel, méthodologique et pratique ${ }^{4}$, il reste que de nombreux obstacles persistent

3 Pour des approfondissements sur ces divers axes de réflexion, nous renvoyons à notre ouvrage : Frédéric Darbellay, Interdisciplinarité et transdisciplinarité en Analyse des Discours. Complexité des textes, intertextualité et transtextualité, Genève, Éditions Slatkine, 2005.

4 Voir notamment pour des états des lieux et synthèses à ce sujet : Werner Arber et al. (Hrsg.), Inter- und Transdisziplinarität, Warum? - Wie?. Inter- et transdisciplinarité, pourquoi? comment?, Berne, Haupt, 1993; Pasqualina 
sur le chemin de sa réalisation effective et qu'il convient de les prendre en compte pour mieux les dépasser. Bien que l'interdisciplinarité soit en effet vivement souhaitée et encouragée par et dans les discours des institutions universitaires, ces dernières restent pourtant massivement organisées en facultés, départements, laboratoires, unités et centres de recherche relativement autonomes. Ce découpage institutionnel permet certes les approfondissements disciplinaires nécessaires à l'avancement des connaissances de pointe, mais il représente dans le même temps une forme de barrière organisationnelle qui peut, selon les cas, freiner voire condamner par avance toute tentative de mise en dialogue des disciplines. Comme le soulignait déjà Gusdorf de manière alarmante dans le contexte mouvementé de 1968 et redoublant de "fureur iconoclaste ${ }^{5}$ " contre le système universitaire :

La situation des universités contemporaines atteste qu'elles ont perdu toute vocation d'universalité. Elles ont cessé d'être des communautés culturelles où se noue l'alliance entre les divers horizons de la connaissance. Plutôt, elles sont devenues des prisons centrales de la culture, soumises à un régime cellulaire, où chacun se retranche à l'abri de cloisons étanches, avec le seul souci de faire valoir son petit domaine à l'abri de toute présence indiscrète. Les universités se sont fragmentées en facultés, en départements, en instituts dont le superbe isolement exclut l'esprit de dialogue. Chaque parcelle de la tunique sans couture de la connaissance se veut exclusive de toutes les autres; chaque aspect se prend pour un tout, et parfois pour le tout. Personne ne parle plus à personne, et d'ailleurs personne ne comprend plus personne, la diver-

Perrig-Chiello et Frédéric Darbellay (dir.), Qu'est-ce que l'interdisciplinarité? Les nouveaux défis de l'enseignement, Lausanne, Éditions Réalités Sociales, 2002; Frédéric Darbellay et Theres Paulsen (dir.), Le défi de l'Inter-et Transdisciplinarité. Concepts, méthodes et pratiques innovantes dans l'enseignement et la recherche, Lausanne, Presses Polytechniques Universitaires Romandes, 2008; Gertrude Hirsch Hadorn et al. (dir.), Handbook of Transdisciplinary Research, Berlin, Springer Verlag, 2008. Robert Frodeman, Julie Thompson Klein, Carl Mitcham (dir.), The Oxford Handbook of Interdisciplinarity, Oxford University Press, 2010; Gloria Origgi et Frédéric Darbellay (dir.), Repenser l'interdisciplinarité, Genève, Éditions Slatkine, 2010.

5 Yves Lenoir, "L'interdisciplinarité : aperçu historique de la genèse d'un concept ", Cahiers de la recherche en éducation, vol. 2, n 2, 1995, p. 248. 
gence des spécialités entraînant la multiplication des langages et des méthodologies ${ }^{6}$.

Gusdorf constatait l'émiettement de la connaissance dans l'organisation des universités, causé par la spécialisation accrue des disciplines et représentant une difficulté de taille pour les essais d'élaboration de connaissances interdisciplinaires. Ce propos coup de poing, sans doute encore vraisemblable à l'heure actuelle alors même que l'on reconnaît que l'université est en mutation ${ }^{7}$, peut être rattaché à une «idéologie interdisciplinaire ${ }^{8}$ » qui lutte ouvertement contre la spécialisation disciplinaire, considérée comme la raison principale de la perte d'un savoir totalisant et unificateur. Dans cette optique, l'institution universitaire est pensée comme une tour de Babel, où les rivalités entre disciplines l'emportent sur l'esprit de dialogue et où les connaissances vives sont malheureusement "incarcérées " et " endisciplinées ${ }^{9}$ ". Alors même que les universités appellent de leurs vœux l'interdisciplinarité et qu'elles laissent d'ailleurs souvent se mettre en place dans leurs murs des initiatives qui vont dans ce sens, on constate que leur structure organisationnelle, que ce soit aux niveaux des contenus des formations, de la gestion des projets de recherche ou du système de promotion des carrières académiques et des publications, tend à reconduire l'hétérogénéité des approches disciplinaires, la polysémie des concepts et la diversité des méthodes. La disciplinarisation décourage, voire bloque, les aspirations des enseignants-chercheurs dans leur volonté de mise en dialogue des connaissances. L'organisation institutionnelle des universités offre finalement un cadre de travail qui, soit pénalise le développement de réflexions interdisciplinaires sous prétexte de l'excellence disciplinaire, soit la recommande et l'encourage

6 Georges Gusdorf, "Interdisciplinarité (connaissance) », Encyclopedia Universalis, vol. 8, Paris, 1968, p. 1088.

7 Voir notamment: Georges Felouzis (dir.), Les mutations actuelles de l'Université, Paris, PUF, 2003; Gilles Breton et Michel Lambert (dir.), Globalisation et universités. Nouvel espace, nouveaux acteurs, UNESCO, Les Presses de l'Université Laval/Economica, 2003.

8 Guy Palmade, Interdisciplinarité et idéologies, Paris, Éditions Anthropos, 1977.

$9 \quad$ Georges Gusdorf, op. cit., 1968. 
tout en restant dans une situation paradoxale où les moyens et les modes de gouvernance peinent à suivre les intentions pourtant louables des responsables institutionnels.

Les obstacles organisationnels à l'interdisciplinarité sont logiquement construits en rapport avec l'histoire fragmentée des sciences, institutionnalisant des rapports hiérarchiques et cloisonnés entre les disciplines académiques. Ces contrariétés institutionnelles sont ainsi également doublées d'" obstacles épistémologiques ${ }^{10}$ ", tant sur le plan de l'histoire du champ scientifique qui s'est constitué par découpages en disciplines et sous-disciplines, que sur celui de la construction différentielle des objets de connaissance disciplinaires et du fonctionnement clôturé des communautés de chercheurs. De manière cumulative, on peut aussi relever des obstacles psycho-sociologiques à la réalisation de l'interdisciplinarité, dans la mesure où l'hétérogénéité des profils spécialisés rejouent le morcellement institutionnel des connaissances sur l'homme et le social, mus par le besoin de reconnaissance académique inhérent au régime parcellaire de la disciplinarité : les esprits disciplinaires ne sont pas à l'abri de la logique mentale du "diviser pour régner ", se confortant parfois dans le rôle du pouvoir symbolique des " tyrannies magistrales ${ }^{11}$ ». Signalons enfin des obstacles culturels, au sens où la disjonction entre de multiples points de vue disciplinaires est parfois renforcée par une séparation entre des aires culturelles, des traditions de pensée, des langues et des mentalités différentes, diversité qui est en l'occurrence souvent perçue comme un obstacle et non comme une potentialité à exploiter en termes d'interdisciplinarité.

Si le système académique génère des connaissances disciplinaires pointues, il engendre par là même une série d'obstacles potentiels sur les plans institutionnel, épistémologique, psychosociologique et culturel. Cette convergence d'obstacles est susceptible de créer les conditions d'une certaine incommunicabilité entre les chercheurs, qui se réclament de paradigmes conceptuels 
et méthodologiques différents, si ce n'est incommensurables. Poussée à l'extrême, la persistance de préjugés et de stéréotypes réciproques entre les spécialistes conduit à une forme d'interincompréhension mutuelle qui ne fait que confirmer des positionnements théoriques et méthodologiques disjoints. Dans ces conditions, toute tentative de dépassement disciplinaire serait perçue comme une intrusion étrangère dans les autres territoires disciplinaires, elle présenterait un caractère de déviance par rapport au modèle académique en vigeur : toute tentative de dépassement serait purement et simplement " assimilée à indiscipline, c'est-à-dire à un manquement à la norme de fait sinon à la règle de droit ${ }^{12}$ ".

La prise en compte des obstacles, parfois bien réels, à la mise en place d'un travail interdisciplinaire montre que l'avènement d'une théorie unifiée de l'interdisciplinarité qui serait partagée par l'ensemble des chercheurs en sciences humaines et sociales est sans doute encore utopique à ce jour. C'est en comprenant par contre la nature de ces obstacles que l'on peut envisager leur dépassement dans et par des pratiques d'enseignement et de recherche interdisciplinaires in vivo ${ }^{13}$. Sans mettre en crise révolutionnaire le système académique existant, il faut sans doute opter pour une dynamique de changement sur fond de continuité, en y aménageant progressivement les adaptations institutionnelles, organisationnelles, épistémologiques et méthodologiques devenues nécessaires. Si l'on se contraint volontairement, compte tenu de l'état encore largement fragmentaire des disciplines constitutives du champ des Sciences Humaines et Sociales, à ne pas construire une théorie générale de l'interdisciplinarité, on peut par contre rassembler des éléments de définition qui permettent d'en dessiner les contours théoriques et conceptuels.

12 Guy-Olivier Faure, "La mise en œuvre de l'interdisciplinarité : barrières institutionnelles et intellectuelles ", dans Eduardo Portella (dir.), Entre savoirs : l'interdisciplinarité en acte : enjeux, obstacles, perspectives, Toulouse, Erès, 1992, p. 110.

13 Dans la présente contribution, nous considérons ensemble les pratiques d'enseignement et de recherche interdisciplinaires, alors même qu'il va de soi que l'interdisciplinarité en général se rejoue aussi de manière relativement spécifique dans les contextes de l'enseignement et de la recherche. 


\section{Définir l'interdisciplinarité : convergences théoriques}

Les réflexions théoriques sur et autour de l'interdisciplinarité témoignent d'une forte polysémie dans les termes utilisés pour désigner ce mode de décloisonnement et d'articulation entre deux ou plusieurs disciplines. On peut aisément repérer une configuration sémantique parfois nébuleuse, où se côtoient les termes de monodisciplinarté, de polydisciplinarité, de métadisciplinarité, de multidisciplinarité, d'interdisciplinarité, ou encore de transdisciplinarité. Il se dégage au moins un point commun de cette multiplicité sémantique : tous les termes s'ancrent sur le concept noyau de disciplinarité, tout en le nuançant par l'usage de divers préfixes (pluri-, uni-, poly-, multi-, mono-, inter-, trans-, etc.) qui reconfigurent successivement et de manière complémentaire l'idée nodale de discipline. Sur le fond de ce réseau sémantique et de manière relativement consensuelle aujourd'hui ${ }^{14}$, les notions-clés suivantes peuvent être mises au premier plan, soit la disciplinarité, la pluri-/multidisciplinarité, l'interdisciplinarité et la transdisciplinarité. D'un point de vue théorique, on postule que ces termes sont susceptibles de s'organiser dans un réseau conceptuel cohérent et en niveaux complémentaires sur et à partir du concept de discipline. On peut ainsi succinctement définir ces niveaux d'interaction entre les disciplines ${ }^{15}$, l'interdisciplinarité prenant sens dans son rapport constitutif et différentiel à la disciplinarité, à la pluri-/multidisciplinarité et à la transdisciplinarité.

\subsection{Disciplinarité}

Du point de vue de la construction des sciences, une discipline se caractérise par une institutionnalisation-standardisation des

\footnotetext{
14 Voir notamment : Jean Piaget, op. cit., 1973; Julie Thompson Klein, Interdisciplinarity. History, Theory, and Practice, Detroit, Wayne State University Press, 1990; Basarab Nicolescu, La transdisciplinarité. Manifeste, Monaco, Éditions du Rocher, 1996; Pasqualina Perrig-Chiello et Frédéric Darbellay (dir.), op.cit., 2002. Frédéric Darbellay, op.cit., 2005; Frédéric Darbellay et Theres Paulsen (dir.), op. cit., 2008.

15 Nous empruntons librement ici à nos propres travaux cités ci-dessus en note infrapaginale.
} 
pratiques de recherche et d'enseignement dans une communauté scientifique donnée ${ }^{16}$, socialement et historiquement située et régie par un paradigme qui définit les présupposés et les objectifs des savoirs à construire. La disciplinarité implique donc la juxtaposition institutionnelle de plusieurs communautés de spécialistes, réparties en autant de facultés, de départements et de laboratoires autonomes. Une discipline est une « catégorie organisationnelle " au sens de Morin :

Elle institue la division et la spécialisation du travail et elle répond à la diversité des domaines que recouvrent les sciences. Bien qu'englobée dans un ensemble scientifique plus vaste, une discipline tend naturellement à l'autonomie, par la délimitation de ses frontières, le langage qu'elle se constitue, les techniques qu'elle est amenée à élaborer ou à utiliser, et éventuellement par les théories qui lui sont propres ${ }^{17}$.

La spécialisation des connaissances, par la démultiplication des approches, des postures épistémologiques, des langages disciplinaires et des méthodes, opère ainsi un découpage fragmentaire de plus en plus poussé des objets d'étude ${ }^{18}$. Ces communautés de spécialistes sont plongées dans un certain isolement académique, bloquant apparemment toute possibilité de dialogue interdisciplinaire. À ce titre, Adam Karpinski et Marcel Samson repèrent les luttes d'influence qui découlent de l'institutionnalisation des disciplines :

Les disciplines ont conservé une attitude de "chasse gardée" qui provient de la division traditionnelle de la connaissance. Les disciplines ont commencé à se livrer des luttes d'influence plutôt que d'essayer d'établir un lien entre elles. Ces luttes d'influence sont survenues avec l'institutionnalisation des disciplines, ce qui a amené des problèmes reliés aux approches conceptuelles différentes, à savoir des problèmes de données, de théories, de méthodologies différentes ${ }^{19}$.

16 Gérard Fourez, La construction des sciences. Les logiques des inventions scientifiques, Bruxelles, De Boeck Université, 2002.

17 Edgar Morin, "Sur l'interdisciplinarité ", dans Carrefour des sciences, Actes du Colloque du Comité National de la Recherche Scientifique ("Interdisciplinarité »), Paris, Éditions du CNRS, 1990, p. 21.

Voir aussi Jacques Hamel, "Réflexions sur l'interdisciplinarité à partir de Foucault, Serres et Granger ", Revue européenne des sciences sociales, Tome XXXIII, n 100, 1995, p. 191-205.

19 Adam Karpinsky et Marcel Samson, "L'interdisciplinarité », Cahiers du 


\subsection{Pluri-/multidisciplinarité}

Dans une perspective pluri- ou multidisciplinaire, un objet d'étude donné ou un problème théorique et/ou pratique à résoudre sont abordés de manière successive et juxtaposée sur la base de deux ou de plusieurs points de vue disciplinaires disjoints, sans véritable interaction entre eux. Ce dispositif confirme en quelque sorte l'institutionnalisation et la standardisation des pratiques d'enseignement et de recherche que nous mentionnons ci-dessus à propos de la disciplinarité. L'approche pluridisciplinaire reste ainsi dans le champ d'attraction de la disciplinarité, elle en reproduit le principe par l'addition-juxtaposition de plusieurs disciplines, sans nécessairement chercher les zones d'interaction entre elles. Comme le remarque en effet Schlanger : « la notion même de discipline implique la pluralité des disciplines [...]. Elle suppose une organisation du savoir qui renonce au rêve totalisant, à l'intuition holistique de l'unité systématique du savoir ${ }^{20}$ ». Un objet de recherche donné, qu'il soit en l'occurrence psychologique, social ou anthropologique, est analysé séquentiellement par une série de disciplines indépendantes, via une opération de découpage-segmentation. Cette simple juxtaposition-addition de disciplines conforte l'hétérogénéité des points de vue sur le(les) objet(s) analysé(s). L'exemple des traditionnels séminaires ou colloques pluridisciplinaires est, à ce titre, tout à fait représentatif : une série de conférences y sont données successivement à un public plutôt spécialisé, les chercheurs s'informant alors mutuellement de l'état d'avancement de leurs travaux autour d'un thème spécifique et transmettant de manière plus ou moins exhaustive leurs résultats d'analyse, sans véritablement interagir entre eux.

\subsection{Interdisciplinarité}

Sur une base pluridisciplinaire, l'interdisciplinarité marque le pas dans la mise en interaction de deux ou de plusieurs disciplines : le préfixe inter- signifie bien ce qui est "entre", soit la relation de

C.R.U.R., nº 2, Montréal, Presses Universitaires du Québec, 1973, p. 17.

20 Judith Schlanger, «Fondation, nouveauté, limites, mémoire », Communications, n 54, Paris, Seuil, 1992, p. 292. 
réciprocité entre plusieurs disciplines dans laquelle on se situe pour décrire, analyser et comprendre la complexité d'un objet d'étude commun. L'interdisciplinarité va au-delà de la simple juxtaposition de plusieurs points de vue disciplinaires, elle vise la collaboration entre spécialistes d'horizons disciplinaires différents et complémentaires, voire l'intégration entre les disciplines. L'interdisciplinarité traduit le "désir d'une connivence élargie ${ }^{21}$ " entre plusieurs champs disciplinaires, elle est fondée sur un partage consenti des objectifs, des méthodes et des langages spécifiques à chacune des disciplines impliquées dans le processus de co-production des connaissances. L'approche interdisciplinaire vise en effet à contrer " l'aveuglement du spécialiste ${ }^{22}$ ", récusant le « caractère territorial du pouvoir par le savoir " pour y substituer un "pouvoir partagé ".

L'interdisciplinarité désigne une façon particulière d'interroger le savoir pour qu'il ne voile pas la vérité sous l'horizon totalitaire de ses acquisitions. Mais elle ne peut réaliser cette tâche que dans une confrontation de plusieurs disciplines à propos ou à partir d'un même objet. En questionnant un même objet d'étude, les spécialistes mettent en question leur discipline et reconnaissent que celle-ci est une façon originale de comprendre et de parler, éprouvée à partir d'une réflexion sur un objet préalablement sélectionné. C'est donc bien la pratique de l'échange interdisciplinaire qui permet une juste appréciation de la discipline sous les deux aspects de la prise de conscience de sa relativité et de la découverte de sa radicale spécificité ${ }^{23}$.

On peut avancer, en suivant Barthes ${ }^{24}$, que la nouveauté de l'interdisciplinarité « ne provient pas forcément du renouvellement intérieur » de chaque discipline prise en elle-même et pour elle-même, mais plutôt « de leur rencontre au niveau d'un objet qui par tradition ne relève d'aucune d'elles ».

$21 \quad$ Ibid., p. 293.

22 Jean-Paul Resweber, La méthode interdisciplinaire, Paris, PUF, 1981, p. 19.

23 Ibid., p. 44.

24 Roland Barthes, "De l'œuvre au texte ", dans Le Bruissement de la langue, Essais critique IV, Paris, Seuil, [1971] 1984, p. 69-77. 
On dirait en effet que l'interdisciplinaire, dont on fait aujourd'hui une valeur forte de la recherche, ne peut s'accomplir par la simple confrontation de savoirs spéciaux; l'interdisciplinaire n'est pas de tout repos : il commence effectivement (et non par la simple émission d'un vœu pieux) lorsque la solidarité des anciennes disciplines se défait, peut-être même violemment à travers les secousses de la mode, au profit d'un objet nouveau, d'un langage nouveau, qui ne sont ni l'un ni l'autre dans le champ des sciences que l'on visait paisiblement à confronter; c'est précisément ce malaise de classification qui permet de diagnostiquer une certaine mutation ${ }^{25}$.

La mise en commun des compétences et des savoirs disciplinaires peut avoir lieu à des niveaux différents d'interaction : il peut s'agir d'opérations de transferts ou d'emprunts de concepts ou de méthodes d'un champ scientifique à l'autre, de mécanismes d'hybridation ou de croisement entre les disciplines, voire la création de nouveaux champs de recherche par couplage de deux ou plusieurs disciplines. Le processus de mise en dialogue des disciplines nécessite que chaque discipline en coprésence mobilise ses compétences et ses outils d'analyse, tout en s'ouvrant aux méthodes des autres disciplines : l'objet de connaissance, complexe et émergent, est ainsi co-construit dans et par le processus interdisciplinaire sur la base des compétences existantes, tout en étant irréductible à l'un ou l'autre des points de vue disciplinaires concernés.

\subsection{Transdisciplinarité}

Bien que nous n'abordions pas de manière spécifique la transdisciplinarité dans les limites de cette contribution, signalons néanmoins qu'elle recouvre différentes orientations complémentaires. Selon une première orientation à visée plus épistémologi$q^{2} e^{26}$, la transdisciplinarité représente un processus de connaissance qui va au-delà (trans-) des frontières disciplinaires. La transdisciplinarité se définit alors comme une vision globale et intégrée,

\footnotetext{
25 Ibid., p. 69.

26 Voir notamment Jean Piaget, op. cit., 1973; Basarab Nicolescu, op. cit., 1996; Denys de Béchillon, "La notion de transdisciplinarité ", dans Guerre et paix entre les sciences. Disciplinarité, inter et transdisciplinarité, La revue du MAUSS semestrielle, $\mathrm{n}^{\circ} 10,1997$, p. 185-200.
} 
qui réorganise les savoirs disciplinaires en vue de la compréhension d'un objet d'étude complexe et « qui ne se contenterait pas d'atteindre des interactions ou des réciprocités entre recherches spécialisées, mais situerait ces liaisons à l'intérieur d'un système total sans frontières stables entre les disciplines ${ }^{27}$ ". Selon une deuxième orientation plus pragmatique, participative et appliquée, la transdisciplinarité peut être pensée comme un processus de recherche qui intègre dans sa propre réalisation les acteurs politiques, sociaux, économiques et citoyens pour la résolution de problèmes complexes (environnement, violence, santé, etc.) : les acteurs externes au domaine scientifique sont amenés à participer activement à la co-construction des connaissances, en partenariat avec les spécialistes du domaine concerné ${ }^{28}$. Il faut enfin relever que la transdisciplinarité s'applique également à l'exploration des relations complexes qui se tissent dans la mise en dialogue entre les cultures scientifiques issues des sciences humaines et sociales, ainsi que des sciences techniques, de la vie et de la nature ${ }^{29}$.

Cette mise au point conceptuelle montre la progressivité et les interrelations entre ces différents paliers de complexification des liens entre les disciplines scientifiques. De la pluri-, à l'inter- et la transdisciplinarité, il y a bien une dynamique sémantique et épistémologique commune qui consiste à baser ces types d'approche sur des ancrages disciplinaires clairement identifiés, tout en les intégrant dans un réseau de relations de plus en plus dense et interactif. On voit comment l'interdisciplinarité se définit par complémentarité et différentiation entre la pluri- et la trans-, et

$27 \quad$ Ibid., p. 8.

28 Voir, par exemple, Gertrude Hirsch Hadorn et al. (dir.), op. cit., 2008. Notons que l'implication des acteurs extrascientifiques n'est pas exclusive à cette version de la transdisciplinarité. Une telle démarche participative est également envisageable dans les cas de multi- et d'interdisciplinarité.

29 Voir notamment : Frédéric Darbellay, Moira Cockell, Jérôme Billotte, Francis Waldvogel (dir.), A Vision of Transdisciplinarity. Laying Foundations for a World Knowledge Dialogue, Lausanne, EPFL Press/CRC Press, 2008;. Moira Cockell, Jérôme Billotte, Frédéric Darbellay et Francis Waldvogel (dir.), Common Knowledge: The Challenge of Transdisciplinarity, Lausanne, EPFL Press/CRC Press, 2011. 
en quoi elle ne correspond en aucune manière à une vision éclectique entre les savoirs disciplinaires, ni à un simple amalgame par addition-juxtaposition. L'interdisciplinarité se distingue de l'organisation disciplinaire des savoirs, mais elle entend néanmoins capitaliser sur les disciplines en les décompartimentalisant. Apprendre à travailler dans l'inter-, l'entre-deux, c'est sans doute renoncer momentanément à construire une théorie unifiée de l'interdisciplinarité qui viendrait simplement homogénéiser le champ des Sciences Humaines et Sociales. Il s'agit de penser dans la diversité, de manière dialogique, pour mieux comprendre la complexité spécifique des objets humains et sociaux, par nature irréductibles à un seul point de vue disciplinaire.

\section{Complexité des objets, multiplicité des points de vue et pensée dialogique}

Les objets d'études des Sciences humaines et sociales, soit l'humain et le social dans leurs multiples dimensions psychologique, anthropologique, historique et culturelle, ont ceci de commun qu'ils demeurent irréductibles à une seule de ces dimensions et qu'ils sont, à ce titre, des objets complexes. Ils constituent « un tissu (complexus : ce qui est tissé ensemble) de constituants hétérogènes inséparablement associés ${ }^{30} »$. La pensée de Pascal raisonne ici comme un signal :

Toutes choses étant causées et causantes, aidées et aidantes, médiates et immédiates, et toutes s'entretenant par un lien naturel et insensible qui lie les plus éloignées et les plus différentes, je tiens impossible de connaitre les parties sans connaitre le tout, non plus que de connaitre le tout sans connaître particulièrement les parties ${ }^{31}$.

L'humain et le social, pensés dans leur globalité, sont plus que la simple somme de leurs constituants bio-psycho-socio-anthroplogico-culturels, ils représentent des entités complexes et émergentes, inscrites dans des contextes et des temporalités sociohistoriques en perpétuel mouvement. Ils participent claire-

\footnotetext{
30 Edgar Morin, Introduction à la pensée complexe, Paris, ESF Éditeur, 1990, p. 21.

31 Cité, entre autres, en exergue dans Edgar Morin (dir.), Relier les connaissances. Le défi du XXI' siècle, Paris, Seuil, 1999.
} 
ment des mondes d'œuvres et de culture au sens de Dilthey ${ }^{32}$ et ils doivent être pensés comme des formes symboliques ${ }^{33}$. Ils sont ainsi emprunts d'une profonde plasticité qui les rend par nature irréductibles à une simple formalisation sous l'angle d'un seul point de vue d'analyse. Les Sciences Humaines et Sociales, ou les Sciences de la culture ${ }^{34}$, sont confrontées au défi de la complexité, dans la mesure où leurs objets d'étude sont hétérogènes et pris dans une dynamique de production du sens dans des contextes de production et de réception sociohistoriquement situés. Les SHS réclament en définitve une "épistémologie de la diversité ${ }^{35}$ ", que l'on peut arrimer à une épistémologie non cartésienne ${ }^{36}$, non dogmatique, non paradigmatique et profondément dialogique, qui inspire tout à la fois une épistémologie de la convergence et une convergence des épistémologies ${ }^{37}$.

Se confronter à la complexité de l'homme et du social, c'est donc dépasser les "piliers de certitude de la science "classique" " (principes d'ordre, de séparation, de réduction et de logique déductive-identitaire $)^{38}$ qui ont eu pour conséquence majeure de dissoudre la complexité des objets d'études (de la particule au macrocosme et de la cellule à l'homme et à la société tout entière) pour la ramener (la réduire) à une simplicité plus maîtrisable. Il faut dépasser les limites de ce paradigme simplificateur de la disjonction-réduction et affronter le défi de la complexité. Penser de manière complexe l'homme et le social, c'est alors se donner les moyens théoriques d'intégrer la variation inhérente à leurs fonctionnements effectifs, ainsi que les relations de circularité et d'interdépendance qui se tissent entre leurs éléments constitutifs. Si le paradigme disjonctif propre à la pensée occidentale

$32 \quad$ Wilhelm Dilthey, Le monde de l'esprit, Paris, Aubier, [1925] 1947.

33 Ernst Cassirer, La philosophie des formes symboliques, 3 vol., Paris, Minuit, [1972] 1975.

34 François Rastier et Simon Bouquet (dir.), Une introduction aux sciences de la culture, Paris, PUF, 2002.

35 François Rastier, Arts et sciences du texte, Paris, PUF, 2001, p. 280.

36 Gaston Bachelard, Le nouvel esprit scientifique, Paris, PUF, 1934.

37 Voir Georges Gusdorf, op.cit., 1968.

38 Edgar Morin et Jean-Louis Le Moigne, L'intelligence de la Complexité, Paris, L'Harmattan, 1999, p. 112. 
moderne a pu poser le principe selon lequel «l'unité ne peut se concevoir qu'en excluant ou en occultant la diversité et vice versa $^{39}$ ", l'approche complexe et systémique est au contraire capable d'associer l'un et le divers " dans une conception fondamentale de l'unitas multiplex ${ }^{40} »$. L'unité et la diversité des objets sociohumains ne s'opposent pas, ceux-ci sont à la fois un et divers, pensés comme des systèmes complexes auto-éco-ré-organisés. La prise en compte de cette complexité réclame une approche qui soit elle-même complexe et interdisciplinaire. C'est parce que les objets sociohumains sont définis comme complexes qu'ils nécessitent l'articulation de plusieurs points de vue disciplinaires pour leur analyse. Cette dialectique entre le sujet et l'objet de connaissance permet d'inverser la fameuse formule de Saussure dans le Cours de linguistique générale, selon laquelle " c'est le point de vue qui crée l'objet ${ }^{41}$ ». Elle n'est en effet que partiellement opérationnelle, dans la mesure où elle implique, réciproquement, la formule inverse et complémentaire : c'est (également/inversement) l'objet qui crée le point de vue.

Aborder le domaine des Sciences Humaines et Sociales sous l'égide de la complexité, c'est se situer dans une oscillation constante entre l'unité et la diversité, entre l'homogénéité et l'hétérongénéité des points de vues qui travaillent dans l'entredeux des disciplines. C'est là que réside la spécificité de l'interdisciplinarité, qui demeure tendue entre une force centripète qui tend au rassemblement du savoir et une force centrifuge ${ }^{42}$ qui tend à disloquer la connaissance. Il en ressort une dialectique structurante dans l'organisation des savoirs et dans leur développement historique : "la division, la spécialisation de l'épistémologie appellent un mouvement compensateur; le vecteur centrifuge doit avoir pour contrepartie un vecteur centripète destiné à

39 Edgar Morin et Massimo Piatelli-Palmarini, «L'unité de l'homme comme fondement et approche interdisciplinaire ", dans Interdisciplinarité et sciences humaines, vol.1, Paris, UNESCO, 1983, p. 194.

40 Ibid.

41 Ferdinand de Saussure, Cours de linguistique générale, Paris, Payot, [1916] 1992, p. 23.

42 Georges Gusdorf, « Passé, présent, avenir de la recherche interdisciplinaire ", Interdisciplinarité et sciences humanies, vol.1, Paris, UNESCO, 1983. 
maintenir la cohésion de l'espace mental, menacé de dissociation $^{43}$ ». Gusdorf avait bien repéré cette oscillation entre unité et diversité, inhérente à l'interdisciplinarité :

Le projet de l'interdisciplinarité dessine d'âge en âge l'un des grands axes de l'histoire de la connaissance. À mesure que la progression du savoir se réalise par spécialisation, le souci de l'unité suscite le désir d'un regroupement qui porterait remède à l'intolérable émiettement des domaines de la connaissance et des hommes de science ${ }^{44}$.

La perspective d'innovation que porte en elle l'interdisciplinarité est sans doute liée à cette dialectique construite entre plusieurs disciplines, qui sont alors prises dans un double processus de fragmentation en domaines spécialisés et de recomposition de ces spécialités en domaines transversaux. Selon Dogan et Pahre, par exemple, «la spécialisation scientifique entraine la fragmentation de disciplines entières en sous-disciplines ", en premier lieu, et en second lieu " cette spécialisation atteignant ses limites naturelles, les chercheurs qui innovent recombinent ces fragments dans des domaines hybrides ${ }^{45}$ ». Dans ce contexte, il n'apparaît pas pertinent de théoriser a priori et de manière figée la question de l'interdisciplinarité; il convient, au contraire, de mettre un principe de pensée dialogique aux commandes de cette réflexion, en contrant les tentatives d'unification théorique au profit d'un processus interdisciplinaire dynamique qui se reconfigure de cas en cas selon les configurations disciplines mises en dialogue.

Suivons le principe dialogique tel que défini par Morin et Le Moigne : "Le principe dialogique unit les deux principes ou notions antagonistes, qui apparemment devraient se repousser l'un l'autre, mais qui sont indissociables et indispensables pour comprendre une même réalitée ${ }^{46} »$. Penser par principe dialogique, c'est ainsi conjoindre les deux visées apparemment antagonistes de l'unité et de la diversité constitutives tant de l'homme et de la société que des multiples disciplines qui les prennent pour

\footnotetext{
43 Ibid., p. 32.

44 Ibid., p. 33.

45 Mattei Dogan et Robert Pahre, L'innovation dans les sciences sociales, la marginalité créatrice, Paris, PUF, 1991, p. 11.

46 Edgar Morin et Jean-Louis Le Moigne, op.cit., 1999, p. 254.
} 
objets d'étude. Le principe dialogique permet d'assumer rationnellement l'association de ces notions contradictoires et il offre une assise épistémologique à partir de laquelle la complexité des objets d'étude peut être abordée : la complexité de l'objet à décrire est mise en corrélation avec une méthodologie et des présupposés épistémologiques eux-mêmes complexes et multidimensionnels. Le dialogisme favorise une nouvelle intelligibilité " allant du Même à l'Autre sans supprimer la différence ${ }^{47}$ ". La prise en compte de la complexité appelle donc une posture interdisciplinaire qui puisse à la fois penser la diversité des disciplines et leur mise en dialogue par un processus de médiation ${ }^{48}$ co-construit par l'interaction entre plusieurs domaines du savoir. Dans la mesure où le travail interdisciplinaire ne ferait volontairement pas l'objet d'une mise en théorie arbitraire, mais qu'il serait lui-même pensé comme une question complexe et plurielle, on peut s'interroger sur la pertinence d'une visée paradigmatique dans ce domaine.

\section{Le paradigme impossible}

Considérant les obstacles à dépasser pour mettre en œuvre l'interdisciplinarité, les acquis de ses contours définitionnels et la pensée complexe et dialogique qu'elle réclame, on peut s'interroger sur la pertinence d'une vision paradigmatique qui tenterait de stabiliser une théorie unifiée de l'interdisciplinarité. En d'autres termes, l'interdisciplinarité peut-elle à ce jour être instaurée comme un (ou le) nouveau paradigme dominant dans le champ des Sciences Humaines et Sociales ou faut-il au contraire plus modestement tenter de construire une nouvelle donne entre les savoirs et les compétences disciplinaires déjà constitués? La notion de paradigme (ou de matrice disciplinaire) forgée par

\footnotetext{
$47 \quad$ Emmanuel Levinas, Noms propres, Montpellier, Fata Morgana, 1976.

48 Jules Duchastel et Danielle Laberge, "La recherche comme espace de médiation interdisciplinaire ", dans L’interdisciplinarité ordinaire. Le problème des disciplines en sciences sociales, Jean-Michel Berthelot, Brigitte Dumas, Luc Racine (dir.), Sociologie et Sociétés, vol. XXXI, nº 1, Montréal, Les Presses de l'Université de Montréal, 1999, p. 63-76.
} 
$\mathrm{Kuhn}^{49}$ se définit comme un certain accord sur les méthodes et les présupposés épistémologiques qui guident à un moment donné la pratique des chercheurs appartenant à une même communauté scientifique. Un paradigme fonde une démarche théorique commune à un groupe de spécialistes à une époque donnée de l'évolution du champ scientifique, il fixe de manière prioritaire le type de problèmes à résoudre au sein de la discipline, les choix méthodologiques partagés, l'utilisation d'un langage spécialisé et l'établissement de procédures unifiantes de validation, de vérification et d'élaboration du travail de recherche. Il s'agit d'une situation de normalisation-normalité ${ }^{50}$ de la science, qui prétend résoudre les problèmes susceptibles d'être posés dans les limites de l'application de méthodes attestées et qui, notons-le avec Bourdieu, "tend à faire oublier qu'elle ne résout que les problèmes qu'elle peut poser ou qu'elle ne pose que les problèmes qu'elle peut résoudre ${ }^{51}$ ".

Si la vision paradigmatique semble pertinente pour décrire une organisation disciplinaire des savoirs en paradigmes ou matrices disciplinaires juxtaposés et incommensurables, elle se révèle par contre inopérante dans le cadre plus ouvert et dialogique de l'interdisciplinarité. La configuration massivement pluridisciplinaire des Sciences Humaines et Sociales est certes une condition nécessaire au travail interdisciplinaire, mais elle programme dans le même temps une difficulté, voire une impossibilité sans doute salutaire, à la mise en paradigme d'une théorie de l'interdisciplinarité. À ce stade, il n'existe pas de science normalisée des pratiques interdisciplinaires, mais plutôt un espace négocié et dialogique plus propice à des controverses méthodologiques productives entre différents points de vue disciplinaires qu'à une phase de crise ou de révolution scientifique. Les réflexions théoriques sur l'interdisciplinarité en SHS ne relèvent ni de

49 Thomas Kuhn, La Structure des révolutions scientifiques, Paris, Flammarion, [1962] 1983.

50 Kuhn parle à ce sujet de science normale et de matrice disciplinaire.

51 Pierre Bourdieu, "La spécificité du champ scientifique et les conditions sociales du progrès de la raison ", Sociologie et Sociétés, vol. VII, n 1,1975 , p. 104. 
"stratégies de conservation-succession ${ }^{52}$ " au sens de Bourdieu, qui viseraient à assurer la perpétuation de l'ordre scientifique établi, ni de "stratégies de subversion " peut-être risquées à ce jour, mais plutôt d'un état intermédiaire de discontinuité sur fond de continuité :

L'antinomie de la rupture et de la continuité s'affaiblit dans un champ qui, ignorant la distinction entre les phases révolutionnaires et la "science normale", trouve dans la rupture continue le vrai principe de sa continuité; et corrélativement l'opposition entre les stratégies de succession et les stratégies de subversion tend de plus en plus à perdre son sens $s^{53}$.

Cette vision plus évolutive et constructive du développement scientifique consiste en définitive à " décrire la science en devenir ou la science qui se fait plutôt que les résultats acquis ${ }^{54}$ ". Face aux cloisonnements persistants des domaines du savoir en Sciences Humaines et Sociales, Piaget signalait déjà la difficulté de faire émerger une pensée partagée, dans la mesure où « chacun répète que l'avenir appartient aux recherches interdisciplinaires, mais, en fait, elles sont souvent très difficiles à organiser à cause d'ignorances réciproques parfois systématiques ${ }^{55}$ ".

\section{Conclusion}

L'interdisciplinarité est prise dans une dialectique entre le besoin de capitaliser sur des compétences disciplinaires acquises et la nécessité de dépasser les cloisonnements inhérents à l’organisation disciplinaire des savoirs, "sans demeurer esclave d'un conservatisme universitaire hérité d'une classification des branches du savoir fondée sur des cloisonnements pédagogiques et de hiérarchies sociales, et qui ne tient pas compte des interactions ou des circularités ${ }^{56}$ ". Face à la doxa disciplinaire bien ancrée dans le domaine des Sciences humaines et sociales et portée par

Ibid., p. 108.

Ibid., p. 108.

Jean Piaget, "Classification des disciplines et connexions interdisciplinaires", Revue internationale des sciences sociales, vol. XVI, n 4, Paris, 1964, p. 598.

55 Ibid., p. 599.

56 Ibid., p. 603. 
ceux que nous pouvons considérer comme " les centraux, les orthodoxes, les continuateurs ", l'interdisciplinarité ouvre un espace de médiation pour les enseignants-chercheurs hétérodoxes, c'est-à-dire "les marginaux, les hérétiques, les novateurs qui se situent souvent aux frontières de leur discipline (qu'ils traversent parfois) ou qui créent de nouvelles disciplines à la frontière de plusieurs champs ${ }^{57}$ ». Comme nous l'avons défendu tout au long de cette contribution, on peut également conclure avec Resweber qu'il existe sans doute peu de " tactiques particulières à l'interdisciplinarité, c'est-à-dire de parcours obligés, balisés et banalisés, en vue de produire un effet de sens calculé ».

Il ne manque pas en revanche de stratégies, c'est-à-dire de parcours à inventer, au fur et à mesure que se déploie l'action interdisciplinaire, de détours à entreprendre pour contourner un obstacle ou pour résoudre une difficulté, de recours à solliciter auprès de disciplines dont on ne mesurait pas l'importance au départ ${ }^{58}$.

Au lieu de prétendre à une théorie unifiée de l'interdisciplinarité, projet d'ailleurs tout à fait légitime, il apparaît qu'il faille en premier lieu créer une culture de tolérance réciproque entre les disciplines, une forme d'empathie (inter-)disciplinaire au sens cognitif de Berthoz ${ }^{59}$, c'est-à-dire la capacité à comprendre le point de vue de l'autre, à se mettre à sa place, mais sans renoncer pour autant à son identité. Il s'agit d'opérer un décentrement qui permette de manipuler plusieurs points de vue complémentaires dans le traitement de l'homme et du social compris dans leur complexité.

57 Pierre Bourdieu, Science de la science et réflexivité, Paris, Éditions Raison d'Agir, 2001, p. 87.

58 Jean-Paul Resweber, op. cit., 1981, p. 19.

59 Alain Berthoz et Gérard Jorland (dir.), L'Empathie, Paris, Odile Jacob, 2005. 


\section{Bibliographie}

Arber, Werner et al. (Hrsg.), Inter- und Transdisziplinarität, Warum? - Wie?. Inter- et transdisciplinarité, pourquoi? comment?, Berne, Haupt, 1993.

Bachelard, Gaston, Le nouvel esprit scientifique, Paris, PUF, 1934.

Barthes, Roland, "De l'œuvre au texte ", dans Le Bruissement de la langue, Essais critique IV, Paris, Seuil, [1971] 1984, p. 69-77.

Béchillon, Denis (de), "La notion de transdisciplinarité ", dans Guerre et paix entre les sciences. Disciplinarité, inter et transdisciplinarité, La revue du MAUSS semestrielle, $n^{\circ} 10,1997$, p. 185-200.

Berthoz, Alain et Gérard Jorland (dir.), L'Empathie, Paris, Odile Jacob, 2005.

Bourdieu, Pierre, "La spécificité du champ scientifique et les conditions sociales du progrès de la raison ", Sociologie et Sociétés, vol. VII, $\mathrm{n}^{\circ} 1$, 1975, p. 91-118.

Bourdieu, Pierre, Science de la science et réflexivité, Paris, Éditions Raison d'Agir, 2001.

Breton, Gilles et Michel Lambert (dir.), Globalisation et universités. Nouvel espace, nouveaux acteurs, UNESCO, Les Presses de l'Université Laval/ Economica, 2003.

Cassirer, Ernst, La philosophie des formes symboliques, 3 vol., Paris, Minuit, [1972] 1975.

Cockell, Moira, Jérôme Billotte, Frédéric Darbellay, Francis Waldvogel (dir.), Common Knowledge: The Challenge of Transdisciplinarity, Lausanne, EPFL Press/CRC Press, 2011.

Darbellay, Frédéric, Interdisciplinarité et transdisciplinarité en Analyse des Discours. Complexité des textes, intertextualité et transtextualité, Genève, Éditions Slatkine, 2005.

Darbellay, Frédéric, Moira Cockell, Jérôme Billotte, Francis Waldvogel (dir.), A Vision of Transdisciplinarity. Laying Foundations for a World Knowledge Dialogue, Lausanne, EPFL Press/CRC Press, 2008.

Darbellay, Frédéric et Theres Paulsen (dir.), Le défi de l'Inter-et Transdisciplinarité. Concepts, méthodes et pratiques innovantes dans l'enseignement et la recherche/Herausforderung Inter- und Transdisziplinarität. Konzepte, Methoden und innovative Umsetzung in Lehre und Forschung, Lausanne, Presses Polytechniques Universitaires Romandes (PPUR), 2008.

Dilthey, Wilhelm, Le monde de l'esprit, Paris, Aubier, [1925] 1947. 
Dogan, Mattei et Robert Pahre, Linnovation dans les sciences sociales, la marginalité créatrice, Paris, PUF, 1991.

Duchastel, Jules et Danielle Laberge, "La recherche comme espace de médiation interdisciplinaire ", dans Jean-Michel Berthelot, Brigitte Dumas, Luc Racine (dir.), L'interdisciplinarité ordinaire. Le problème des disciplines en sciences sociales, Sociologie et Sociétés, vol. XXXI, $\mathrm{n}^{\circ} 1$, Montréal, Les Presses de l'Université de Montréal, 1999, p. 63-76.

Faure, Guy-Olivier, "La mise en œuvre de l'interdisciplinarité : barrières institutionnelles et intellectuelles ", dans Eduardo Portella (dir.), Entre savoirs : l'interdisciplinarité en acte : enjeux, obstacles, perspectives, Toulouse, Erès, 1992, p. 109-116.

Felouzis, Georges (dir.), Les mutations actuelles de l'Université, Paris, PUF, 2003.

Fourez, Gérard, La construction des sciences. Les logiques des inventions scientifiques, Bruxelles, De Boeck Université, 2002.

Frodeman, Robert, Thompson Klein, Julie, Mitcham, Carl (dir.), The Oxford Handbook of Interdisciplinarity, Oxford University Press, 2010.

Gusdorf, Georges, "Interdisciplinarité (connaissance) ", Encyclopedia Universalis, vol. 8, Paris, 1968, p. 1086-1090.

Gusdorf, Georges, "Passé, présent, avenir de la recherche interdisciplinaire ", Interdisciplinarité et sciences humanies, vol. 1, Paris, UNESCO, 1983, p. 31-51.

Hamel, Jacques, "Réflexions sur l'interdisciplinarité à partir de Foucault, Serres et Granger ", Revue européenne des sciences sociales, Tome XXXIII, $\mathrm{n}^{\circ} 100,1995$, p. 191-205.

Hirsch Hadorn, Gertrude et al. (dir.), Handbook of Transdisciplinary Research, Berlin, Springer Verlag, 2008.

Karpinsky, Adam et Marcel Samson, "L'interdisciplinarité ", Cahiers du C.R.U.R., nº 2, Montréal, Presses Universitaires du Québec, 1973.

Kuhn, Thomas, La Structure des révolutions scientifiques, Paris, Flammarion, 1962/1983.

Lenoir, Yves, "L'interdisciplinarité : aperçu historique de la genèse d'un concept ", Cahiers de la recherche en éducation, vol. 2, $\mathrm{n}^{\circ}$ 2, 1995, p. $227-265$.

Levinas, Emmanuel, Noms propres, Montpellier, Fata Morgana, 1976.

Morin, Edgar, «Sur l'interdisciplinarité », dans Carrefour des sciences, Actes du Colloque du Comité National de la Recherche Scientifique ("Interdisciplinarité »), Paris, Éditions du CNRS, 1990, p. 21-29. 
Morin, Edgar, Introduction à la pensée complexe, Paris, ESF Éditeur, 1990.

Morin, Edgar (dir.), Relier les connaissances. Le défi du XXI siècle, Paris, Seuil, 1999.

Morin, Edgar et Jean-Louis Le Moigne, Lintelligence de la Complexité, Paris, L'Harmattan, 1999.

Morin, Edgar et Massimo Piatelli-Palmarini, «L'unité de l'homme comme fondement et approche interdisciplinaire ", dans Interdisciplinarité et sciences humaines, vol. 1, Paris, UNESCO, 1983, p. 191-215.

Nicolescu, Basarab, La transdisciplinarité. Manifeste, Monaco, Éditions du Rocher, 1996.

Origgi, Gloria et Frédéric Darbellay (dir.), Repenser linterdisciplinarité, Genève, Éditions Slatkine, 2010.

Palmade, Guy, Interdisciplinarité et idéologies, Paris, Éditions Anthropos, 1977.

Perrig-Chiello, Pasqualina et Frédéric Darbellay (dir.), Qu'est-ce que l'interdisciplinarité? Les nouveaux défis de l'enseignement, Lausanne, Éditions Réalités Sociales, 2002.

Piaget, Jean, "Classification des disciplines et connexions interdisciplinaires ", Revue internationale des sciences sociales, vol. XVI, n 4, Paris, 1964, p. 598-616.

Piaget, Jean, "L'épistémologie des relations interdisciplinaires », Bulletin Uni-information, $\mathrm{n}^{\circ}$ 31, Genève, 1973, p. 4-8.

Rastier, François, Arts et sciences du texte, Paris, PUF, 2001.

Rastier, François et Simon Bouquet (dir.), Une introduction aux sciences de la culture, Paris, PUF, 2002.

Resweber, Jean-Paul, La méthode interdisciplinaire, Paris, PUF, 1981.

Saussure, Ferdinand (de), Cours de linguistique générale, Paris, Payot, [1916] 1992.

Schlanger, Judith, "Fondation, nouveauté, limites, mémoire ", Communications, ${ }^{\circ}$ 54, Paris, Seuil, 1992, p. 289-298.

Thompson Klein, Julie, Interdisciplinarity. History, Theory, and Practice, Detroit, Wayne State University Press, 1990. 